\title{
Cambios en la tenencia de la tierra en un pueblo de la precordillera del Norte de Chile: Socoroma*
}

\author{
Milka Castro \\ Miguel Babamondes
}

\section{INTRODUCCIÓN}

La organización socioeconómica andina descansa en una forma de producción donde el control del territorio productivo, las relaciones sociales, políticas y de producción adquieren especificidad. Fuentes históricas han testimoniado cómo la necesidad de articulación de las comunidades andinas con la sociedad global fue generando diversas formas de utilización de la mano de obra ind ígena y explotación de los recursos naturales, transformando la base socioeconómica local.

En el extremo norte de Chile, Región de Tarapacá, el acelerado proceso de urbanización que experimenta la ciudad de Arica, a partir de 1952, producto de la liberación de franquicias aduaneras, trajo consigo un drenaje de población y la consolidación de una corriente migratoria permanente de la población aymara desde la precordillera y altiplano ariqueño, atraidos por el mercado de trabajo y de productos, pero también como consecuencia de la internalización de valores de la sociedad occidental; sin duda, un papel relevante juegan en este aspecto la educación formal y otros "atractivos" del modo de vida en la ciudad.

En este trabajo se intenta mostrar cómo una comunidad andina, con un alto índice migratorio, resuelve el control sobre su espacio productivo.

A comienzos de siglo se producía un incremento sostenido de la población del altiplano, precordillera y valles de Arica, situación que se revierte bruscamente a partir de 1952 (cuadro $\mathrm{N}^{\circ} 1$ ).

*Estudio realizado con el apoyo del Departamento de Investigación y Bibliotecas de la Universidad de Chile y de UNESCO-MAB-6. 


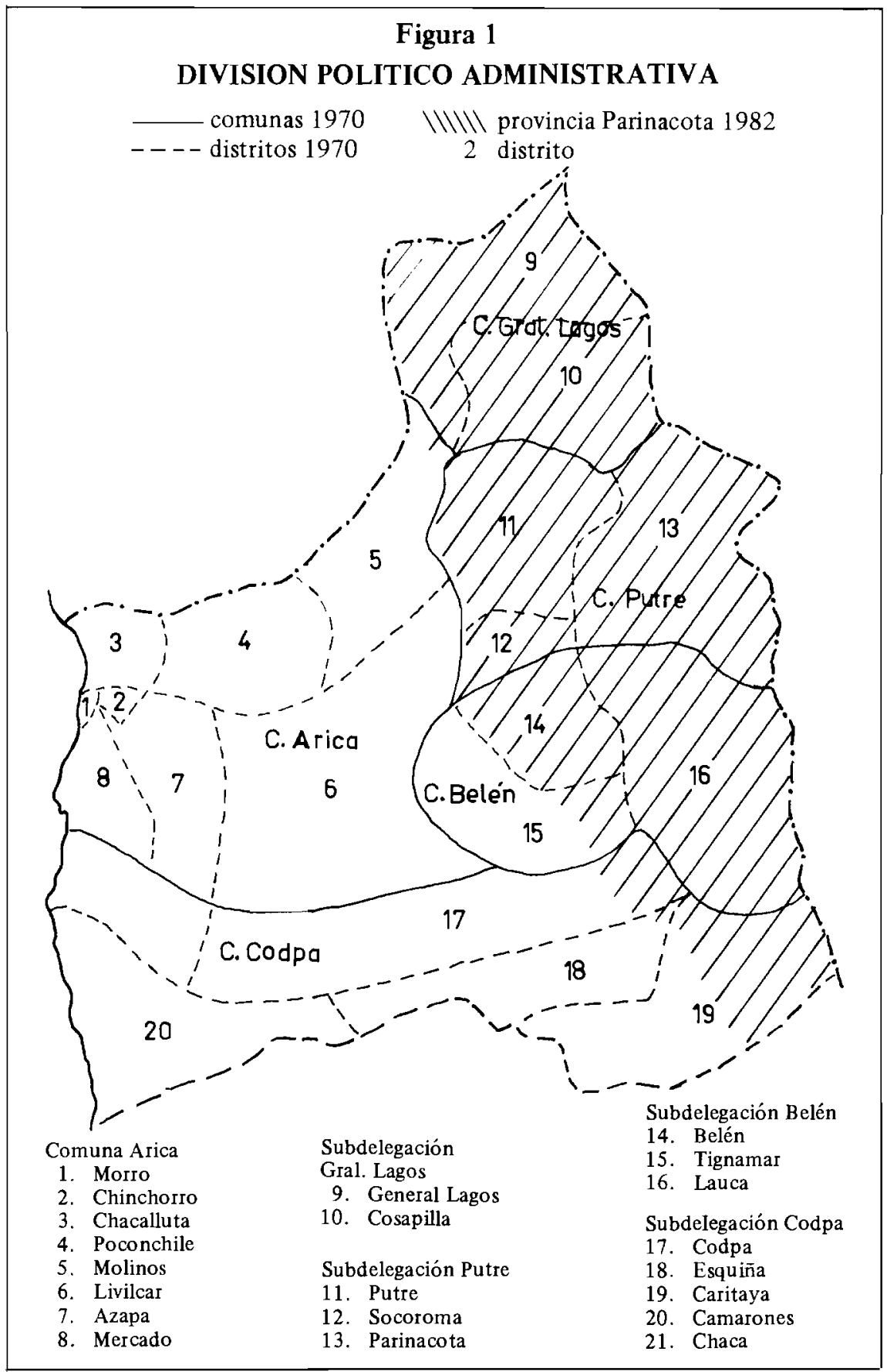

Milka Castro 


\section{Cuadro No 1}

\begin{tabular}{lccccc}
\hline Año & \multicolumn{2}{c}{ Comuna Arica } & & \multicolumn{2}{c}{ Comunas del interior } \\
\cline { 2 - 3 } \cline { 5 - 6 } & Población & $\begin{array}{c}\% \\
\text { variación }\end{array}$ & & Población & $\begin{array}{c}\% \\
\text { variación }\end{array}$ \\
\hline 1920 & 12.463 & & & 4.223 & \\
1930 & 15.912 & $+27,6$ & & 4.373 & $+3,5$ \\
1940 & 16.627 & $+4,5$ & & 5.209 & $+19,0$ \\
1952 & 23.033 & $+38,5$ & & 7.274 & $+40,0$ \\
1960 & 46.686 & $+102,7$ & & 5.261 & $-28,0$ \\
1970 & 92.002 & $+97,1$ & & 3.943 & $-25,0$ \\
1982 & 146.677 & $+59,4$ & & 5.897 & $+50,0$ \\
\hline
\end{tabular}

El aumento de la población del interior que se observa en 1982 requiere de un análisis especial. Por una parte, la creación de la Provincia de Parinacota cambió la estructura político administrativa (Figura 1) concentrando una mayor población. Otro incremento que eleva esta cifra se produce en la parte alta del valle de Lluta ${ }^{1}$.

${ }^{1}$ La mayoría de las veces que se utilizan cifras censales para un análisis demográfico de grupos étnicos se está expuesto a llegar a conclusiones erróneas. Por ello, con la intención de explicar las diferencias de población rural en la zona ariqueña, señalaremos, al menos, dos aspectos: 1) la Comuna de Arica estaba conformada por algunos sectores rurales como es el caso de Molinos y Livilcar en 1970; Chaca fue incorporado en 1982, de acuerdo a la nueva división político-administrativa. Este distrito pertenecía, en 1970, a la subdelegación Codpa. 2) Con la nueva estructura aparecen comunas con una considerable población, tal es el caso de la nueva Comuna de Camarones conformada casi exclusivamente con los distritos que en 1970 pertenecían a la desaparecida subdelegación Codpa. Lo mismo ocurre con Putre; a esta comuna se incorporó pueblos como Belén, Tignamar y Lauca, pertenecientes en 1970 a la subdelegación Belén.

Cuadro comparativo de la División Político-Administrativa: 1970-1982 (Población rural)

\begin{tabular}{|c|c|c|c|c|c|}
\hline 1970 & & & 1982 & & \\
\hline Provincia Arica & $\begin{array}{c}\text { Distri- } \\
\text { tos }\end{array}$ & $\begin{array}{c}\text { Pobla- } \\
\text { ción }\end{array}$ & Provincia Arica & $\begin{array}{c}\text { Distri- } \\
\text { tos }\end{array}$ & $\begin{array}{c}\text { Pobla- } \\
\text { ción }\end{array}$ \\
\hline Comuna Arica (rural) & & 386 & Comuna Arica (rural) & & 735 \\
\hline Comuna subdelegación & & & Comuna Camarones & 5 & 729 \\
\hline General Lagos & 2 & 833 & & & \\
\hline Comuna subdelegación & & & & & \\
\hline Putre & 3 & 846 & Provincia Parinacota & & \\
\hline Comuna subdelegación & & & Comuna Putre & 9 & 3.350 \\
\hline Belén & 3 & 948 & Comuna General & & \\
\hline Comuna subdelegación & & & Lagos & 4 & 1.083 \\
\hline Codpa & 5 & 930 & & & \\
\hline
\end{tabular}

Total población rural

3.943

5.897

Fuente: INE. 
El presente trabajo parte del siguiente enunciado: a pesar de la existencia de un notable proceso migratorio, la superficie de tierra bajo cultivo se mantiene estable. El abandono de algunos sectores cultivables obedecería a escasez de agua.

La actual organización de los factores de la producción constituye el objetivo de estudio de una investigación mayor de la cual este trabajo es sólo parte. Aquí se discutirá en torno a las formas de acceso, tenencia y transferencia de uno de los factores de la producción: la tierra.

La información procede de dos fuentes: a) mapas y registros de propiedad contenidos en documentos de Bienes Nacionales; y b) censos e información primaria que entrega el trabajo de campo realizado por los autores en Socoroma entre los años 1983 y 1988. Mediante un estudio comparativo del listado de predios y propietarios en este poblado de la precordillera, para los años 1947 y 1982, se obtuvo antecedentes sobre los procedimientos de traspaso de los predios, el papel que en ello juega el sexo y parentesco, y la tendencia a la división y concentración de la tierra.

\section{ANTECEDENTES}

Al analizar el período que va de la mitad del presente siglo desde una perspectiva de las acciones de la sociedad mayor, se comprueba que recién a partir de 1959 los gobiernos de Chile empezaron a visualizar el subdesarrollo progresivo del sector andino como problema relevante por sus graves consecuencias demográficas y geopolíticas (VAN KESSEL, 1980: 27). Se inicia entonces una serie de programas: ampliación de caminos, asistencia y equipamiento con tecnología urbana, organización de cooperativas, énfasis en la educación (de mínima competencia con la identidad cultural andina) y proyectos centrados en explotaciones extractivas y exportadoras. Estas acciones, que aceleraron el proceso de transculturación, llevan a Van Kessel, basándose en Vargas (1978), a definir la centenaria política chilena del 'Desarrollo Andino de Tarapacá' como una forma de colonialismo interno (ibíd. 29).

(Cont. de nota 1)

El mayor aumento de población aparece en la Comuna de Putre. Cabe señalar que el pueblo de Putre tiene 1.954 habitantes (1.650 hombres y 304 mujeres), y sólo 263 viviendas. Si comparamos este pueblo de la precordillera con otro del mismo sector, Belén, que posee 235 viviendas y sólo 368 habitantes, y si consideramos que Putre como capital de la nueva provincia de Parinacota ha incorporado un notable número de personal administrativo y militar, podemos suponer que el mayor porcentaje de población no corresponde a habitantes de origen andino. Otro aumento notable que se observa en el cuadro, ocurre en los poblados rurales incorporados en la comuna de Arica, ellos corresponden a valles bajos que, como es el caso de Lluta, se han poblado con personas de origen andino provenientes de Arica o de pisos más altos. Sin pretender agotar las variables que inciden en el análisis explicativo señalaremos, por último, que la mayoría de los distritos de la comuna de Putre disminuyeron su población, y lo mismo ocurre con Codpa. Por ello, tendemos a sostener que la población se ha mantenido en una cifra cercana a la existente según el censo de 1970 . 
En la zona de este estudio, provincias de Arica y Parinacota, los efectos de estas políticas corroboran la opinión vertida por Van Kessel; y en esto también coincidimos con Platt (1975: 35) cuando, refiriéndose a Arica, señala que "la participación en la economía nacional de mercado ha aumentado dramáticamente desde que empezó el crecimiento moderno de la ciudad a fines de la década del 50".

Es por tanto, en este contexto donde debemos situar a Socoroma, poblado de la precordillera ariqueña localizado a una altura aproximada de 3.300 m.s.n.m. (18 $15^{\circ}$, L.S.) en el sistema hidrográfico del río Lluta (Figura 2).

Si bien este trabajo se centra en el estudio de la tenencia de la tierra en el área que circunda inmediatamente al poblado de Socoroma, es necesario referirse brevemente al control ejercido sobre recursos localizados en otras áreas.

Los relatos orales señalan que en tiempos de la fundación de Socoroma no más de seis "familias" tenían derecho reconocido sobre la tierra ${ }^{2}$. Las alianzas matrimoniales y la herencia constituían los principales medios para acceder a la tierra; la utilización de otros medios como apropiación, compra, donación y préstamo existía aunque en forma incipiente y controlada por la comunidad ${ }^{3}$. Era de este modo como se lograba el control espacial dentro y fuera de la comunidad, estrategia fundamental para.superar las limitaciones que impone el medio al hombre andino.

Dentro del sistema hidrográfico Lluta, el control espacial tiende a ser compartido por los pueblos asentados en la precordillera. Específicamente, Putre y Socoroma se adjudican derechos sobre las tierras altas del valle costero de Lluta, hasta la localidad de Molinos, a una distancia aproximada de $35 \mathrm{~km}$ de la costa. La mayoría de las familias de Socoroma declaran haber poseido o poseer tierras fuera del pueblo; en primer lugar figura Lluta, le sigue Zapahuira, Putre, Murmuntani, Epispacha y Chusmiza, todas son quebradas menores de la precordi-

\footnotetext{
${ }^{2}$ En el trabajo de campo se mencionó como "antiguos dueños de Socoroma" a cinco apellidos: Gutiérrez, Vásquez, Loredo, Carrasco y Humire. La presencia de familias con mayor control sobre los recursos se corrobora cuando se ha señalado que a comienzos del presente siglo había un número semejante de "principales del pueblo", y a su muerte se habría producido un proceso de subdivisión y traspaso de la propiedad (por venta o arriendo a quienes habían trabajado para esas familias) como consecuencia de la migración de los herederos (CASTro, M. y BAHAMONDES, M., trabajo inédito).

${ }^{3}$ Como una forma de acceder a la tierra que habría estado vigente hasta mediados de siglo, se ha señalado el reparto de las tierras de los padres en vida, pero mientras éstos vivían, los recién casados debían permanecer un tiempo con ellos, luego la comunidad acordaba darles terrenos suficientes "para subsistir" (uno o dos andenes); podía ser donación de sus padres o arrendamiento, en este caso se preferían terrenos que no estaban siendo trbajados por sus dueños, cancelando en trabajo, productos o dinero. En esta situación podían permanecer un año, luego deb ían comenzar a "cumplir cargos" (alguacil, alférez, mayordomo) como requisito para obtener derecho al agua. El tener hijos era otra condición para acceder al agua de riego. Con posterioridad al cumplimiento de estas obligaciones podían comenzar a arrendar o comprar más tierras.
} 


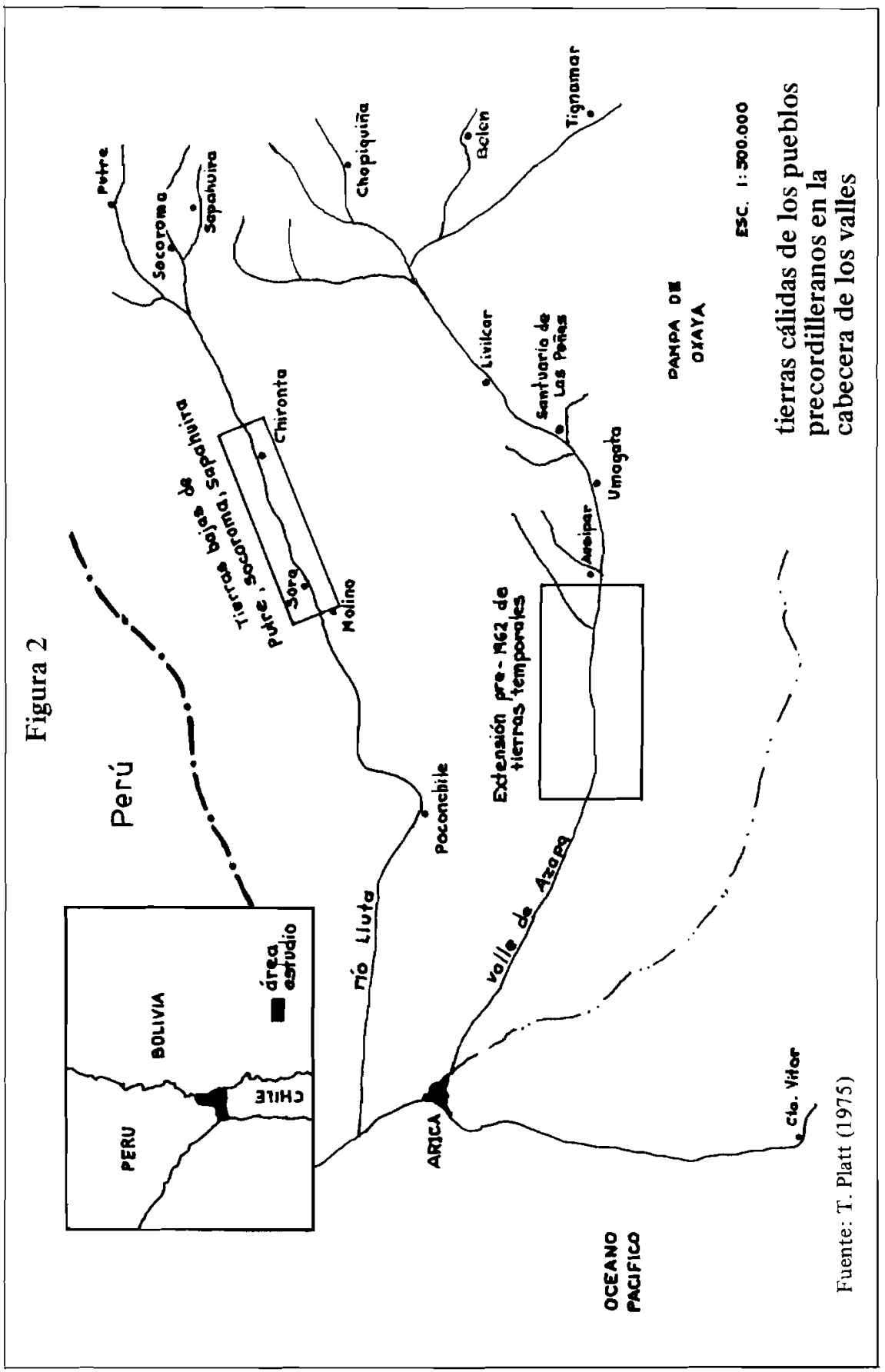


llera. Una situación semejante es descrita por Platt (op. cit. 4l) para el valle de Azapa (Figura 2). "Muchos campesinos de Livilcar, Chapiquiña, Belén y Tignamar acostumbran a bajar cada año en la época de las crecidas a cultivar en el sector entre Cerro Moreno y Ausipar".

El habitante de Socoroma reconoce haber dejado de cultivar directamente, como se hacía hasta una década atrás, sus predios en tierras bajas ${ }^{4}$; hoy son los hijos, preferentemente, quienes asentados en el mismo valle de Lluta o en la ciudad de Arica explotan estos terrenos. Otros han entregado sus tierras en arriendo o mediería; algunos propietarios han vendido $\mathrm{y}$, finalmente, quienes admiten haberlas abandonado. En el momento de la investigación todas las familias establecidas en la parte más alta del valle de Lluta procedían de Socoroma ${ }^{5}$.

A pesar de que no es objetivo de este trabajo profundizar en las formas de complementariedad interecológica de la economía andina, no podemos dejar de señalar, en términos muy generales, que frente a las modificaciones de las formas para acceder a tierras fuera de la comunidad, surgen nuevos patrones de organización socioeconómica que permiten la continuidad en la explotación de este recurso. La complementariedad se ha adaptado a un nuevo modelo: los residentes de Socoroma mantienen la vigencia de las relaciones de intercambio de productos con familiares radicados en Arica o Lluta, y los residentes en la ciudad, que mantienen la propiedad sobre las tierras en Socoroma, acuden periódicamente a trabajar sus predios para obtener, preferentemente, productos para el autoconsumo de la familia en Arica.

\section{SOCOROMA}

Las características ecológicas del área altiplánica comprendida entre $\operatorname{los} 18^{\circ}$ y $23^{\circ}$ L.S. condicionaron el asentamiento de la población en valles y quebradas donde la explotación de los recursos adquirió una cierta uniformidad. Socoroma constituye uno de estos asentamientos prealtiplánicos. El pueblo está conformado por 125 viviendas que se distribuyen en un trazado de calles irregulares alrededor de una iglesia

\footnotetext{
4 "Antiguamente" las quebradas de Zapahuira y Murmuntani pertenecieron a Socoroma, donde sus habitantes debían cumplir cargos. A Lluta los viajes se efectuaban por dos o tres días, de acuerdo a las demandas del trabajo que se iniciaba con la siembra (maíz y alfalfa). En noviembre se cosechaba la alfalfa, oportunidad que se aprovechaba para llevar el ganado ovino y alimentarlo con el rastrojo. Además del trabajo agrícola y pastoreo existían relaciones de intercambio económico. Hasta hoy se utiliza la semilla de alfalfa y el maíz "amarillo" de Lluta.

${ }^{5}$ En el plano religioso, las relaciones entre la población establecida en Lluta y los residentes en Socoroma, se han vuelto conflictivas. Los residentes en Lluta anhelan fervorosamente recuperar la Virgen Candelaria que, con posterioridad a una desastrosa avenida del río, fue rescatada y llevada a Socoroma. Por otra parte, los residentes en Socoroma reclaman derechos sobre tierras que sus abuelos donaron a la iglesia que existía en Sora, y que hoy estarían en manos de particulares.
} 
y plaza centrales. La economía de la comunidad es fundamentalmente agrícola-ganadera (ovinos y bovinos); antiguas construcciones de andenes, constantemente reparados, y dos acequias matrices que bordean las partes altas de los cerros conforman la base del sistema de cultivos en laderas de cerros (maíz, papas, orégano, alfalfa en mayor proporción y algo de horticultura) en una superficie potencialmente productiva cercana a las 300 hectáreas. Esta superficie no se encuentra cultivada en su totalidad, ya sea a raíz de la escasez de agua como a la práctica agrícola de dejar en descanso el terreno.

En este trabajo se estudia un área de aproximadamente 110 hectáreas, que está siendo cultivada, y donde la propiedad privada tiene reconocimiento legal. Dos grandes zonas agrícolas no han sido incluidas por razones diferentes: una, llamada Viscachune, área de tierras no cultivadas hace 7 años debido a que el agua de la acequia que la riega se destina a otro sector. La otra corresponde al Cerro Aroma, frente al pueblo en dirección norte, destinada casi exclusivamente a cultivos anuales (papas); en esta zona la propiedad no tiene reconocimiento legal; sin embargo, cada familia o persona reconoce los límites de su propiedad por derecho consuetudinario (Figura 3).

De las 125 construcciones ya mencionadas, 60 corresponden a viviendas ocupadas en forma permanente, 34 tienen uso temporal, y 25 no se ocupan e incluso se desconoce el nombre del dueño; el resto corresponde a negocios, iglesias y otros (fuente Precenso 1982). El fenómeno migratorio ha llevado a mantener una forma de residencia temporal, es decir, ocupación de la vivienda durante los días en que se debe efectuar el trabajo que demanda la agricultura. También ha determinado una estructura familiar peculiar, puesto que la mayor parte de los miembros de la familia - en el caso de residencia permanente - han migrado y, como se observa en el siguiente cuadro, el $70 \%$ corresponde a familias con una o dos personas por vivienda.

Cuadro $\mathrm{N}^{\circ} 2$

FAMILIAS CON RESIDENCIA PERMANENTE

\begin{tabular}{cccc}
\hline $\begin{array}{c}\text { Miembros por } \\
\text { familia }\end{array}$ & $\begin{array}{c}\text { Número de } \\
\text { familias }\end{array}$ & $\%$ & $\begin{array}{c}\text { Total } \\
\text { personas }\end{array}$ \\
\hline 1 & 24 & 40,0 & 24 \\
2 & 18 & 30,0 & 36 \\
3 & 5 & 8,3 & 15 \\
4 & 5 & 8,3 & 20 \\
5 & 5 & 8,3 & 25 \\
6 & 1 & 1,6 & 6 \\
7 & 2 & 3,3 & 14 \\
\hline TOTAL & $\mathbf{6 0}$ & $\mathbf{1 0 0 , 0}$ & $\mathbf{1 4 0}$ \\
\hline
\end{tabular}




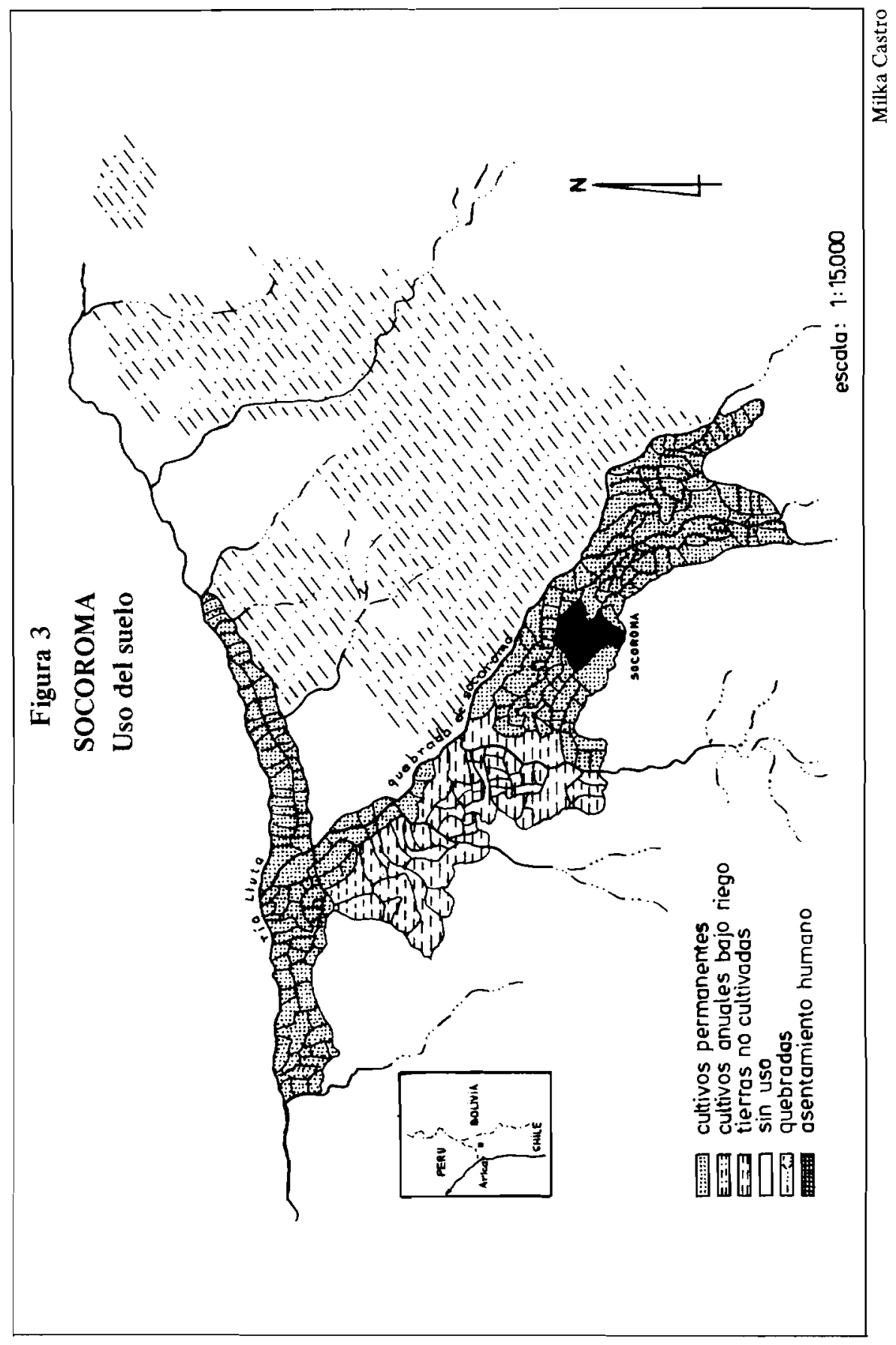


La población residente desciende en un $67 \%$ en los últimos 50 años; las cifras censales señalan que en 1940 Socoroma poseía 430 habitantes y en 1982 posee sólo 140. De hecho, a esta última cifra, para efectos de conocer la organización de la producción, habría que agregar la población que acude mensualmente a trabajar en la producción agrícola. Las personas que permanecen en Socoroma son mayoritariamente de edad avanzada; aquellos matrimonios con hijos en edad escolar prefieren la educación impartida en Arica, donde tienen, además, la posibilidad de continuar estudios en la educación superior. En un análisis de los registros escolares se constató que desde 1920 a 1985 el promedio de matrícula ha descendido desde aproximadamente 70 alumnos por año en la primera mitad del siglo a sólo 10 en los últimos 5 años (1981-1985) (Figura 4). El descenso de la población implica cambios en la estructura demográfica, pero también en la estructura laboral, dado que la forma de producción en Socoroma se basaba en la división del trabajo por sexo y edad. (CASTro, M. y BAHAMONDES, M., trabajo inédito).

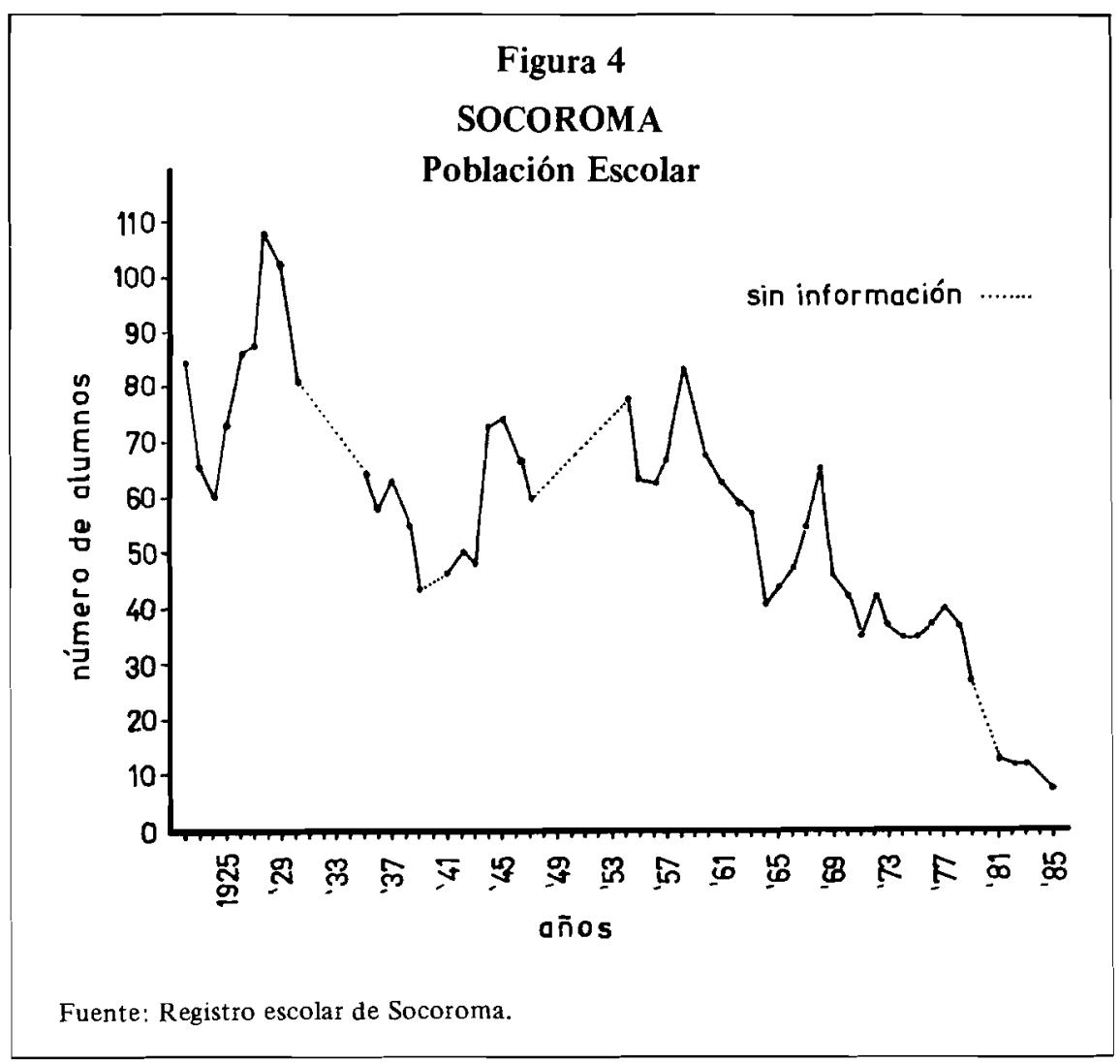


Resultados

La superficie analizada, de acuerdo a la información disponible, presenta las siguientes características ${ }^{6}$ :

\section{Cuadro No 3}

\begin{tabular}{lcccccc}
\hline & \multicolumn{2}{c}{1947} & & \multicolumn{2}{c}{$\mathbf{1 9 8 2}$} \\
\cline { 2 - 3 } \cline { 5 - 6 } & $\begin{array}{c}\text { Cantidad } \\
\text { predios }\end{array}$ & $\%$ & & $\begin{array}{c}\text { Cantidad } \\
\text { predios }\end{array}$ & $\%$ \\
\hline Con información & 465 & 86,91 & & 540 & 95,23 \\
Sin superficie & 15 & 2,80 & & 15 & 2,64 \\
Sin nombre propietario & 45 & 8,41 & & 7 & 1,23 \\
Fiscales & 2 & 0,37 & & 2 & 0,35 \\
Eriazos & 8 & 1,49 & & 3 & 0,52 \\
\hline TOTAL & $\mathbf{5 3 5}$ & $\mathbf{9 9 , 9 8}$ & & $\mathbf{5 6 7}$ & $\mathbf{9 9 , 9 7}$ \\
\hline
\end{tabular}

Como se señala en el cuadro $\mathrm{N}^{\circ} 3$ se debió trabajar con 465 predios para 1947 y con 540 para el año 1982. El aumento de 75 predios es producto de la subdivisión de 38 predios con las siguientes características: 24 lo hacen en dos, dando origen a 48 nuevos predios, y 7 se dividen en tres, dando origen a 21 nuevos predios. La ampliación de la frontera agrícola es casi nula.

En el siguiente cuadro se muestra como, a pesar del fenómeno migratorio descrito, existe, como vimos, un aumento en el número de predios y también un aumento de propietarios donde el comportamiento por sexo es notoriamente distinto. Existe un aumento absoluto en el número de propietarios: el $25 \%$ en el caso de los hombres y $56,4 \%$ en el caso de las mujeres. En términos relativos se produjo un aumento en el número de mujeres propietarias y una disminución de los hombres; no obstante, éstos controlan una mayor cantidad de predios (cuadro $\mathrm{N}^{\circ} 4$ ).

\footnotetext{
${ }^{6}$ Hemos denominado predio a cada terreno que posee un propietario individualizado legalmente. En Socoroma, un andén, llamado "contra", constituye la unidad mínima de propiedad. La propiedad sobre estos andenes adquiere dos modalidades:1) concentración en manos de un solo dueño de todos los terrenos escalonados y que forman una franja delimitada, y 2) posesión de varios dueños de una de las franjas de la pendiente. Esta modalidad se presenta en cada uno de los sectores agroecológicos en que la comunidad ha dividido el espacio productivo. Por tanto, al hablar de división de la propiedad, se está haciendo referencia a un proceso de "disgregación" de la propiedad, dando origen a predios menores constituidos por uno o más andenes llamados "retazos".
} 


\section{Cuadro No 4}

\begin{tabular}{|c|c|c|c|c|c|c|c|c|}
\hline & \multicolumn{4}{|c|}{1947} & \multicolumn{4}{|c|}{1982} \\
\hline & \multicolumn{2}{|c|}{ Propietarios } & \multicolumn{2}{|c|}{ Predios } & \multicolumn{2}{|c|}{ Propietarios } & \multicolumn{2}{|c|}{ Predios } \\
\hline & $\mathbf{N}^{\mathbf{O}}$ & $\%$ & $\mathrm{~N}^{0}$ & $\%$ & $\mathbf{N}^{\mathbf{0}}$ & $\%$ & $\mathbf{N}^{\mathbf{o}}$ & $\%$ \\
\hline Hombres & 88 & 58,67 & 290 & 62,37 & 110 & 53,14 & 309 & 57,22 \\
\hline Mujeres & 62 & 41,33 & 175 & 37,63 & 97 & 46,86 & 231 & 42,78 \\
\hline TOTAL & 150 & 100,00 & 465 & 100,00 & 207 & 100,00 & 540 & 100,00 \\
\hline
\end{tabular}

Teniendo presente, por una parte, el aumento del número de propietarios, el relativamente bajo porcentaje de división física predial $(7,1 \%)$ a lo cual debe incorporarse como dato relevante la casi nula ampliación de la frontera agrícola, $y$, por otra, si se considera lo señalado anteriormente respecto a la propiedad (ver nota 6), podemos sostener que en Socoroma los nuevos predios son producto de la disgregación de algunas propiedades.

A objeto de analizar las características de la transferencia y el papel del sexo y parentesco en ella, se trabajó con 483 predios que poseían la información requerida: nombre del propietario, sexo y parentesco. Así se pudo establecer las siguientes características para las 483 propiedades:

1. 211 predios continúan en poder de su dueño $(43,68 \%)$. De éstos, 147 en propiedad de hombres $(69,7 \%)$, y 64 en propiedad de mujeres $(30,3 \%)$.

2. 272 predios cambian de dueño $(56,31 \%)$, con las siguientes características respecto al papel del sexo:

-153 predios son transferidos por hombres, de los cuales 75 son adquiridos por hombres y 78 por mujeres.

-119 predios son transferidos por mujeres, de éstos 62 son adquiridos por mujeres y 57 por hombres.

3. Respecto del tipo de transferencia sólo se obtuvo información para

19 casos, con las siguientes características:

- 9 predios fueron comprados por hombres

- 10 predios fueron comprados por mujeres

- 12 predios fueron vendidos por hombres

- 7 predios fueron vendidos por mujeres

- 4 predios fueron vendidos a parientes

-15 predios fueron vendidos a no parientes. 
4. En el proceso de transferencia surgen 295 casos, pudiéndose constatar lo siguiente:

-200 predios fueron transferidos a familiares

-93 predios fueron transferidos a no familiares

-2 predios aparecen como propiedad fiscal.

De lo expuesto se puede observar que de las 295 propiedades que cambian de dueño, $200(67,8 \%)$ son transferidas a parientes. Respecto al comportamiento por sexo, si bien el porcentaje de hombres que conservan sus predios es mayor, en el proceso de transferencia las mujeres muestran una inclinación mayor a la adquisición de terrenos en Socoroma.

No obstante la limitada información ( $7 \%$ ) que se obtuvo respecto a la venta de la propiedad se pudo constatar que en el $79 \%$ de los casos fueron adquiridas por no parientes.

Una característica de la conformación de la propiedad agrícola es estar constituida por más de un predio. Al analizar el tamaño de éstos se tiene una variación entre $40 \mathrm{~m}^{2}$ y $38.000 \mathrm{~m}^{2}$ (Figura 5). Comparando los dos años estudiados se observa que en 1947 el $50,6 \%$ de los predios tienen superficies inferiores a los $1.500 \mathrm{~m}^{2}$; en 1982 el porcentaje de predios en ese estrato es de un $56,6 \%$. E1 $93,0 \%$ de los nuevos predios se incorporaron al rango señalado.

En el cuadro 5 se presentan los resultados obtenidos de la comparación efectuada entre la superficie total de las propied ades agrícolas y el número de propietarios. De los socoromeños que en 1947 aparecen como propietarios de tierras, el $56,6 \%$ de ellos controlaban propiedades agrícolas con superficies inferiores a $5.000 \mathrm{~m}^{2}(0,5 \mathrm{Ha})$; en 1982 en esta situación se encuentra el $69,5 \%$ de los casos. (Anexo 1 ).

\section{Cuadro No 5}

\begin{tabular}{rrrrrr}
\hline & \multicolumn{2}{c}{1947} & & \multicolumn{2}{c}{1982} \\
\cline { 2 - 3 } \cline { 5 - 6 } Superficie $\left(\mathbf{m}^{2}\right)$ & Propietarios & $\%$ & & Propietarios & $\%$ \\
\hline $1-5.000$ & 85 & 56,67 & & 114 & 69,57 \\
$5.001-10.000$ & 31 & 20,67 & & 35 & 16,81 \\
$10.001-15.000$ & 21 & 14,00 & & 17 & 8,21 \\
$15.001-20.000$ & 7 & 4,67 & & 6 & 2,90 \\
$20.001-25.000$ & 1 & 0,67 & & 1 & 0,48 \\
$25.001-30.000$ & 2 & 1,33 & & 3 & 1,45 \\
$30.001-35.000$ & $\ldots$ & - & & 1 & 0,48 \\
$35.001-y$ más & 3 & 2,00 & & - & - \\
\hline
\end{tabular}




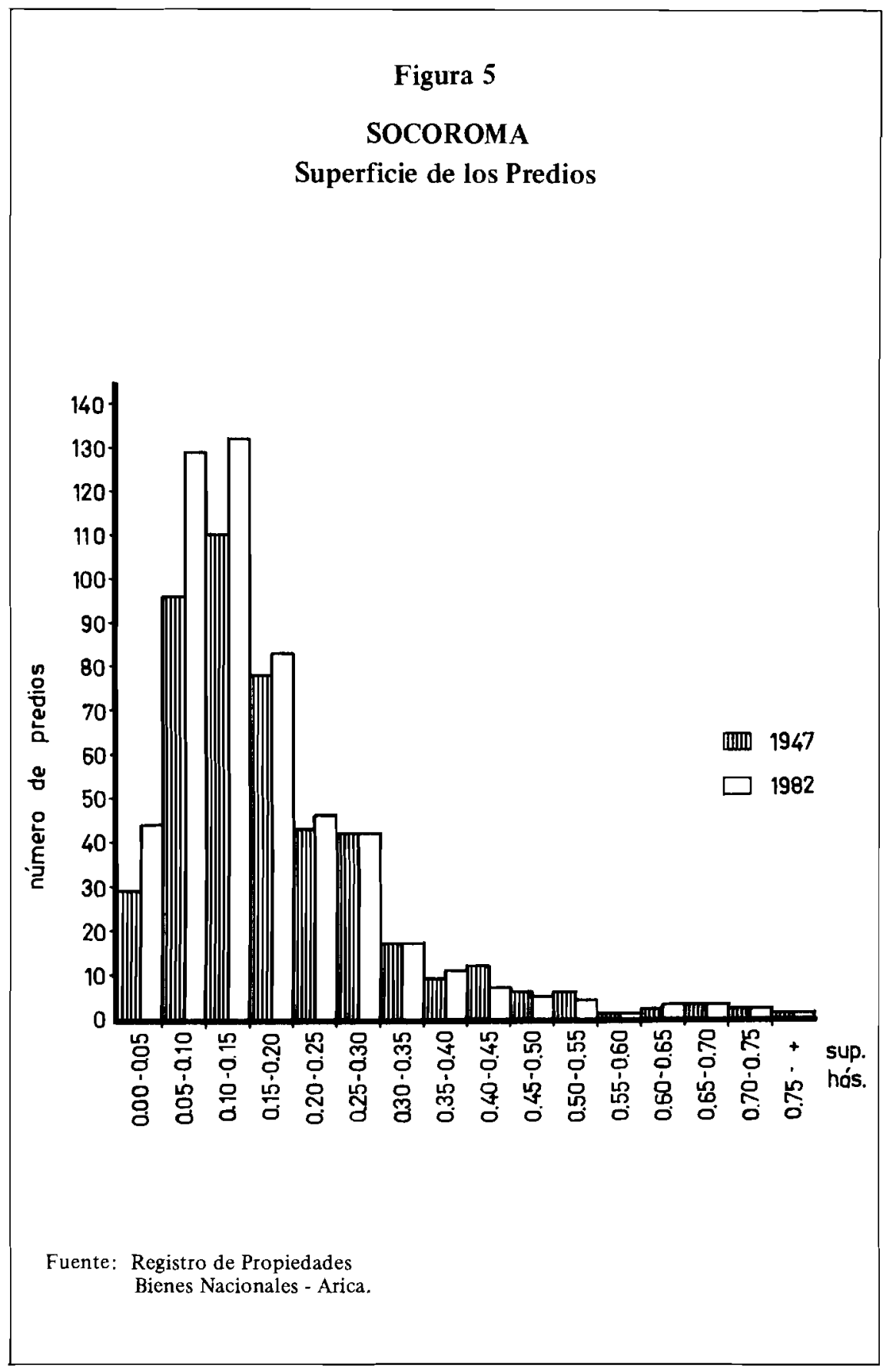


Como señaláramos anteriormente una caracterítica importante en la forma de producción andina ha sido poseer más de un predio o "retazos". En el Cuadro $N^{\circ} 6$ se ha procedido a comparar el número de propiedades en 1947 y 1982; como se puede observar hubo un incremento notable de las personas que poseen entre 1 y 2 propiedades $(65,22 \%)$, y una disminución de propietarios de más de 5 predios.

\begin{tabular}{|c|c|c|c|c|}
\hline \multirow[b]{3}{*}{ Cantidad predios } & \multicolumn{2}{|c|}{ Cuadro No 6} & \\
\hline & \multicolumn{2}{|c|}{1947} & \multicolumn{2}{|c|}{1982} \\
\hline & Propietarios & $\%$ & Propietarios & $\%$ \\
\hline 1 & 56 & 37,33 & 83 & 40,10 \\
\hline 2 & 26 & 17,33 & 52 & 25,12 \\
\hline 3 & 20 & 13,33 & 21 & 10,14 \\
\hline 4 & 13 & 8,67 & 19 & 9,18 \\
\hline 5 & 11 & 7,33 & 15 & 7,25 \\
\hline 6 & 9 & 6,00 & 5 & 2,42 \\
\hline 7 & 6 & 4,00 & 1 & 0,48 \\
\hline 8 & 5 & 3,33 & 4 & 1,93 \\
\hline 9 & - & - & 1 & 0,48 \\
\hline 10 & - & - & 2 & 0,97 \\
\hline 11 & - & -- & 3 & 1,45 \\
\hline 12 & 1 & 0,67 & 1 & 0,48 \\
\hline 13 & 1 & 0,67 & - & 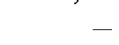 \\
\hline 14 & 1 & 0,67 & - & - \\
\hline 15 & 1 & 0,67 & - & - \\
\hline TOTAL & 150 & 100,00 & 207 & 100,00 \\
\hline
\end{tabular}

En el Cuadro $\mathrm{N}^{\circ} 7$ se muestra un seguimiento a 150 propietarios (comparando año 1947-1982) con la finalidad de conocer las variaciones en la cantidad de predios poseídos por ellos.

Cuadro No 7

\begin{tabular}{lcc}
\hline & Propietarios & $\%$ \\
\hline Conservan & 34 & 22,6 \\
Aumentan & 21 & 14,0 \\
Disminuyen & 40 & 26,6 \\
Pierden & 55 & 36,6 \\
\hline TOTAL & $\mathbf{1 5 0}$ & $\mathbf{9 9 , 8}$ \\
\hline
\end{tabular}


La tendencia observada hacia la disgregación de la propiedad puede ser contrarrestada por la existencia de diversos mecanismos para acceder a la tierra: arriendo, préstamo y mediería; en el siguiente cuadro se quiere destacar que el matrimonio constituye una instancia en que se vuelve a concentrar la propiedad; puesto que tanto el hombre como la mujer aportan tierras, también es posible incorporarlas posteriormente. El Cuadro $\mathrm{N}^{\circ} 8$ muestra en forma resumida los resultados del análisis de 26 matrimonios de los que se disponía de infcrmación para ambos cónyuges; se observa que tanto el hombre como la mujer aumentaron sus predios, pero se destaca el aumento en las propiedades de mujeres.

\section{Cuadro No 8}

Matrimonios

Cantidad de predios

1947

1982

$\%$ aumento

Muj. Homb. Total Muj. Homb. Total Muj. Homb.

\begin{tabular}{lllllllll}
26 & 35 & 87 & 122 & 81 & 113 & 194 & 131,0 & 29,9 \\
\hline
\end{tabular}

Al establecer una comparación entre residentes permanentes y temporales, de acuerdo a la información disponible para 87 casos, se obtuvieron los siguientes resultados:

\section{Cuadro No 9}

\begin{tabular}{lcccccc}
\hline & \multicolumn{3}{c}{ Productores residentes } & & \multicolumn{3}{c}{ Productores temporales } \\
& No & Sup. $\mathbf{m}^{2}$ & $\overline{\mathbf{X}}$ & No $^{\mathbf{2}}$ & Sup. $\mathbf{m}^{2}$ & $\overline{\mathbf{X}}$ \\
\hline Hombres & 31 & 295.038 & 9.517 & 14 & 92.673 & 6.620 \\
Mujeres & 33 & 183.284 & 5.554 & 9 & 54.940 & 6.104 \\
\hline TOTAL & $\mathbf{6 4}$ & $\mathbf{4 7 8 . 3 1 2}$ & $\mathbf{7 . 4 7 3}$ & $\mathbf{2 3}$ & $\mathbf{1 4 7 . 6 1 3}$ & $\mathbf{6 . 4 1 7}$ \\
\hline
\end{tabular}

Atendiendo a las cifras totales no existe una mayor diferencia entre la superficie controlada por cada uno de los sectores; pero al efectuar la distinción por sexo se puede comprobar que existe una escasa diferencia entre la superficie promedio que controlan hombres y mujeres del sector que acude a Socoroma a trabajar las tierras desde Arica, es decir, temporalmente, situación diferente a la de los productores con residencia permanente; entre éstos existe una mayor proporción de tierra en manos de los hombres. 
A objeto de establecer una relación entre los dos tipos de productores señalados y la preferencia por determinados cultivos, se analizaron 61 casos para los que se dispuso de información. Los resultados se han resumido en el cuadro $\mathrm{N}^{\circ} 10$.

Si bien la jerarquización de los cultivos es similar en ambos sectores, se debe prestar atención al hecho de que en los productores temporales es mayor la importancia dada al cultivo de maíz y papas; de igual modo que la producción de alfalfa lo es para los productores con residencia permanente.

Cuadro $\mathrm{N}^{0} 10$

\begin{tabular}{lcccc}
\hline & $\begin{array}{c}\text { Productores } \\
\text { (37 casos) }\end{array}$ & $\begin{array}{c}\text { Residencia } \\
\text { permanente }\end{array}$ & $\begin{array}{c}\text { Productores } \\
\text { (24 casos) }\end{array}$ & $\begin{array}{c}\text { Residencia } \\
\text { temporal }\end{array}$ \\
Cultivos & $\mathbf{N}^{\mathbf{0}}$ predios & $\%$ & $\mathbf{N}^{\mathbf{0}}$ predios & $\%$ \\
\hline Alfalfa & 75 & 41,2 & 28 & 35,4 \\
Orégano & 62 & 34,0 & 26 & 32,9 \\
Maíz & 26 & 14,2 & 13 & 16,4 \\
Papas & 9 & 4,9 & 9 & 11,3 \\
Chacra & 3 & 0,6 & 1 & 1,2 \\
Frutales & 1 & 0,5 & 2 & 2,5 \\
Otros & 6 & 3,2 & 0 & 0,0 \\
\hline TOTAL & $\mathbf{1 8 2}$ & $\mathbf{9 8 , 6}$ & 79 & $\mathbf{9 9 , 7}$ \\
\hline
\end{tabular}

Por último, se ha procedido a analizar la concentración de la propiedad y su relación con el parentesco. En Socoroma, como se ha señalado, se reconocen cinco apellidos principales: Vásquez, Gutiérrez, Loredo, Carrasco y Humire ${ }^{7}$; se procedió a listarlos y establecer una estratificación considerando la superficie y el número de predios (Anexo 2). En 1982 el 15\% de los apellidos - seis de un total de cuarenta- poseen el $55,9 \%$ de la superficie; junto a los siete apellidos siguientes - de la jerarquización hecha de acuerdo a la superficie controlada - el porcentaje se eleva a un $81,9 \%$. Por lo tanto, los 27 apellidos restantes sólo poseen el $17,7 \%$ de la superficie explotada. Esta información no explica la tenencia individual; en los trece apellidos queda comprendido el $74 \%$ de los propietarios.

\footnotetext{
${ }^{7}$ En la Revisita de 1750 a los Altos de Arica, efectuada por el Oficial Real, don Joaquín Cárdenas (HIDALGO, 1978: 159-171), aparecen en Socoroma con mayor frecuencia los siguientes apellidos: Mamani, Limachi, Manglia (¿Manlla?), Umiri, Gutierrez, Condori, Flores, Sisa y Guanca en el mismo orden de importancia. Limachi, Condori y Sisa ya no se encuentran en Socoroma. Hemos destacado los apellidos que aún son representativos. Anexo 2 .
} 


\section{CONSIDERACIONES FINALES}

Refiriéndose al contexto ecológico adverso que asedia al agricultor, Camino sostiene que "se ha caracterizado el sistema agrícola tradicional andino como una estrategia orientada hacia la minimización de riesgos $y$, por tanto, encaminada a garantizar la seguridad en el abastecimiento de alimentos" (1982: 28).

El control diversificado de zonas ecológicas se ha considerado como un componente "ideal" de la ideología andina. Pero hasta dónde es posible que persista este ideal de control cuando las comunidades inevitablemente se enfrentan a la necesidad de "articularse" 8 a los mercados de tierras, bienes y servicios y de trabajo. En Socoroma la situación es clara; el proceso de articulación se ha dinamizado en las últimas décadas y a pesar que mantiene, en líneas gruesas, el ideal andino de control de tierras, se están produciendo cambios en la base de la economía estrechamente ligados a las nuevas formas de vida de la población.

La asignación de títulos de propiedad privada de la tierra y la presión sobre el recurso se traduce en una tendencia donde la subdivisión de la propiedad parece inevitable. Frente a esta situación la familia andina despliega esfuerzos locales para ampliar la propiedad y/o explotación a través de alianzas matrimoniales, compras, préstamos, arriendos y aparcerías de la tierra, mecanismos que cuentan con un reconocimiento y regulación comunal. En la transferencia de la propiedad a terceros se han establecido restricciones locales: se debe dar preferencia a parientes y residentes en Socoroma. Al respecto, en opinión de Sánchez Parga, mientras el factor tierra constituya el principal recurso de supervivencia y reproducción de las familias, las alianzas matrimoniales y la misma estructura de parentesco se organizarán en función del espacio productivo de la comunidad (1984: 184). En el universo de representaciones del mundo andino, la identificación de la tierra con los ancestros se traduce en lazos sociales pasados y presentes que subyacen a la producción (ibíd.: 155).

La posibilidad de acceder a un solo terreno por parte de los matrimonios jóvenes, el cumplimiento de "cargos" religiosos como requisito para disponer de agua de riego, entre otros factores, permitían a los habitantes de Socoroma ejercer un control comunal sobre los recursos. En la actualidad, la asignación de "turnos" de riego como una atribución de la Junta de Vecinos; el otorgamiento de títulos de propiedad privada individual, lo que acarrea consigo la liberación del

\footnotetext{
${ }^{8}$ González de Olarte $(1984: 22)$ distingue articulación e integración como dos categorías distintas. "La primera se refiere a los distintos mecanismos de circulación de mercancías, de fuerza de trabajo y de capital, a través de los cuales los comuneros se relacionan con otros productores. Mientras que integración se refiere al efecto duradero que tiene esta articulación sobre la producción, distribución de recursos e ingresos y el consumo, y las relaciones sociales en las comunidades y entre los comuneros".
} 
mercado de la tierra, y el creciente carácter comercial de los principales cultivos, se transforman en poderosos mecanismos que presionan por descomunalizar el control sobre áreas de cultivo. En opinión de Mayer, esto está en directa relación con el grado de mercantilización: "a mayor mercantilización de productos, mayor presión de descomunalizar los controles de una zona; a mayor producción para el uso directo, mayor será la presión por mantener los controles comunales" (1977: 69).

Lo anterior es posible constatarlo en la comunidad teniendo presente dos fenómenos directamente relacionados. Por una parte, si bien es cierto que en Socoroma existe una fuerte tendencia a la subdivisión de la propiedad, ello no puede llevarnos a no considerar el proceso inverso: la concentración de la tierra. La información permite constatar la existencia de familias que han incrementado el número de predios controlados por ellas. Por otra parte, el orégano, cultivo eminentemente comercial, ocupa aproximadamente el $34 \%$ de la superficie cultivada.

A lo ya expresado, es posible incorporar otra variable que contribuye a explicar la diferenciación. Como se señalara en un comienzo, en la zona andina las comunidades efectúan una división y explotación del recurso tierra teniendo presente las condiciones agroecológicas, y la información de campo ha registrado el reconocimiento por parte de los habitantes de Socoroma de sectores en el lugar más aptos para uno u otro cultivo; se puede concluir que el proceso de división de la propiedad, junto al de acumulación, contribuye a acentuar la diferenciación al interior de la comunidad. La diferencia no tan sólo es resultado de contar con mayor o menor superficie para desarrollar actividades agropecuarias, sino que también se encuentra asociada a la posibilidad de controlar tierras en diferentes lugares a los cuales se les reconoce una cierta especialización productiva.

En el control de tierras desde la ciudad de Arica, la fuerza de trabajo que aporta la mujer es fundamental; mientras el resto de su familia se incorpora a la educación o trabajo asalariado, la mujer acude periódicamente a efectuar las labores que demanda el ciclo agrícola. Este hecho, contribuye, por otra parte, a explicar la importancia que ha adquirido la mujer en el control sobre la tierra.

Para concluir, podemos señalar que las tendencias que se observan en la reestructuración de la tenencia de la tierra en Socoroma, particularmente en lo que dice relación con la acumulación individual, al igual que en otras comunidades andinas (Durston, 1983: 113), se sustenta en instituciones y sistemas valóricos que en otro contexto sirvieron para asegurar el autoconsumo familiar y comunal. En opinión de Matos Mar (1979:30), "el incremento de la propiedad individual de las tierras de cultivo, su escasez, la secuela de un sector desposeido, la progresiva pauperización de sus tierras y la consecuente migración... tienden a descomponer la organización social comunal". Aunque estas afirmaciones parecen verdaderas, no es menos cierto que la población 
andina lleva siglos de adaptación y, si bien actualmente enfrenta un proceso de cambios muy acelerado, producto de la "modernización" y "privatización" de la tierra, las estrategias de organización socioeconómica persisten.

\section{Bibliografía}

Camino, Alejandro. "Tiempo y espacio en la Estrategla de Subsistencia Andina: Un caso en las Vertientes Orientales Sudperuanas", en Senri Ethonological Studies, El hombre y su ambiente en los Andes Centrales (Osaka), $\mathrm{N}^{\circ} 10$, pp. $11-35,1982$.

Castro, Milka y Bahamondes, Miguel. "Acceso y control de los recursos en una comunidad andina: Socoroma", M.S.

Durston, John. "Comunidad andina, gestión de recursos y diferenciación social", en Sobrevivencia campesina en ecosistema de altura, CEPAL-PNUMA. Santiago, Vol. I, pp. 91-137, 1983.

González, Efrain. La economia de la comunidad andina, Lima, Instituto Estudios Peruanos, 1984.

Hidalgo, Jorge. Revisita a los Altos de Arica en 1750, Arica, Departamento de Antropología, Universidad del Norte, 1978.

Matos Mar, José. "Comunidades indigenas del área andina", A nuario Indigenista, Vol. XXXVII, diciembre, pp. 9-35, 1977.

Mayer, Enrique. "Tenencia y control comunal de la tierra: caso de Laraos (Yanyos)", Cuadernos del Consejo Nacional de la Universidad Peruana, (Lima), pp. 59-71, enero-junio, 1977.

Platt, Tristán. "Experien cia y experimentación: Los asentamientos andinos en las cabeceras del Valle de Azapa", Chungará (Arica), $\mathrm{N}^{\circ}$ 5, pp. 33-60, 1975.

Sánchez, José. "Estructuras espaciales del parentesco en los Andes: Salamalagchico", en Estrategias de supervivencia de la comunidad andina, Quito, pp. 154-216, 1984.

Van Kessel, Juan. Holocausto al progreso. Los aymaras de Tarapacá, Amsterdam, CEDLA Incidentele Publicaties 16, 1980. 
Anexo 1

TAMAÑO DE LAS PROPIEDADES AGRICOLAS Y NUMERO DE PROPIETARIOS

\begin{tabular}{|c|c|c|c|c|c|}
\hline \multirow{2}{*}{$\begin{array}{l}\text { Superficie } \\
\left(\text { metros }^{2}\right)\end{array}$} & \multicolumn{2}{|c|}{1947} & \multicolumn{2}{|l|}{1982} & \multirow{2}{*}{$\begin{array}{c}\text { Cambios } \\
\% \\
\end{array}$} \\
\hline & Propietarios & $\%$ & Propietarios & $\%$ & \\
\hline $1 \cdot 1.000$ & 12 & 8,00 & 21 & 10,14 & $2,14+$ \\
\hline $1.001-2.000$ & 30 & 20,00 & 55 & 26,57 & $6,57+$ \\
\hline $2.001-3.000$ & 19 & 12,66 & 32 & 15,45 & $2,79+$ \\
\hline $3.001 \cdot 4.000$ & 14 & 9,33 & 22 & 10,62 & $1,29+$ \\
\hline $4.001-5.000$ & 10 & 6,66 & 14 & 6,76 & $0,10+$ \\
\hline $5.001 \cdot 6.000$ & 9 & 6,00 & 5 & 2,41 & $3,59-$ \\
\hline $6.001 \cdot 7.000$ & 6 & 4,00 & 6 & 2,89 & 1,11 \\
\hline $7.001-8.000$ & 8 & 5,33 & 10 & 4,83 & $0,50-$ \\
\hline $8.001 \cdot 9.000$ & 5 & 3,33 & 7 & 3,38 & $0,05+$ \\
\hline $9.001-10.000$ & 3 & 2,00 & 7 & 3,38 & $1,38+$ \\
\hline $10.001-11.000$ & 5 & 3,33 & 7 & 3,38 & $0,05+$ \\
\hline $11.001 \cdot 12.000$ & 5 & 3,33 & 4 & 1,93 & $1,40-$ \\
\hline $12.001 \cdot 13.000$ & 7 & 4,66 & 3 & 1,44 & 3,22 \\
\hline $13.001-14.000$ & 2 & 1,33 & 1 & 0,48 & 0,85 \\
\hline $14.001-15.000$ & 2 & 1,33 & 2 & 0,96 & $0,37 \ldots$ \\
\hline $15.001-16.000$ & 2 & 1,33 & 2 & 0,96 & 0,37 \\
\hline $16.001 \cdot 17.000$ & 3 & 2,00 & 1 & 0,48 & 1,52 \\
\hline $17.001-18.000$ & 1 & 0,66 & 2 & 0,96 & $0,30+$ \\
\hline $18.001-19.000$ & 1 & 0,66 & - & - & 0,66 \\
\hline $19.001 \cdot 20.000$ & - & $\ldots$ & 1 & 0,48 & $0,48+$ \\
\hline $20.001-21.000$ & - & -- & - & - & - \\
\hline $21.001 \cdot 22.000$ & - & - & - & - & - \\
\hline $22.001 \cdot 23.000$ & - & -- & - & - & - \\
\hline $23.001-24.000$ & - & - & 1 & 0,48 & $0,48+$ \\
\hline $24.001 \cdot 25.000$ & 1 & 0,66 & - & - & 0,66 \\
\hline $25.001-26.000$ & - & - & 1 & 0,48 & $0,48+$ \\
\hline $26.001-27.000$ & 1 & 0,66 & 2 & 0,96 & $0,30+$ \\
\hline $27.001-28.000$ & 1 & 0,66 & - & - & 0,66 \\
\hline $28.001 \cdot 29.000$ & - & -- & - & - & - \\
\hline $29.001 \cdot 30.000$ & - & - & - & - & - \\
\hline $30.001 \cdot 31.000$ & - & -- & - & - & - \\
\hline $31.001-32.000$ & - & - & - & - & - \\
\hline $32.001-33.000$ & - & - & - & - & - \\
\hline $33.001-34.000$ & - & -- & - & - & - \\
\hline $34.001-35.000$ & - & - & 1 & 0,48 & $0,48+$ \\
\hline $35.001-36.000$ & 1 & 0,66 & - & - & 0,66 \\
\hline 36.001 y más & 2 & 1,33 & - & - & 1,33 \\
\hline TOTAL & 150 & & 207 & & \\
\hline
\end{tabular}


Anexo 2

\begin{tabular}{|c|c|c|c|c|c|}
\hline \multirow[b]{2}{*}{ Apellidos } & \multirow[b]{2}{*}{ Predios } & \multicolumn{4}{|c|}{ Año: 1947} \\
\hline & & $\%$ & Sup. $\left(m^{2}\right)$ & $\%$ & Propietarios \\
\hline Vásquez & 68 & 14,6 & 112.290 & 11,17 & 12 \\
\hline Humire & 59 & 12,7 & 165.742 & 16,46 & 12 \\
\hline Flores & 54 & 11,6 & 81.736 & 8,20 & 12 \\
\hline Huanca & 43 & 9,2 & 76.701 & 7,70 & 14 \\
\hline Gutiérrez & 40 & 8,6 & 74.082 & 7,44 & 12 \\
\hline Carrasco & 30 & 6,4 & 59.333 & 5,95 & 12 \\
\hline Bolaños & 17 & 3,6 & 38.890 & 3,90 & 8 \\
\hline Guarache & 16 & 3,4 & 34.475 & 3,46 & 6 \\
\hline Mamani & 14 & 3,0 & 101.445 & 10,18 & 9 \\
\hline Alanoca & 13 & 2,8 & 34.849 & 3,50 & 4 \\
\hline Gómez & 12 & 2,5 & 30.050 & 3,01 & 3 \\
\hline Manlla & 12 & 2,5 & 23.482 & 2,35 & 4 \\
\hline Choque & 11 & 2,3 & 18.063 & 1,81 & 3 \\
\hline Fernández & 9 & 1,9 & 22.256 & 2,23 & 5 \\
\hline Quispe & 8 & 1,7 & 9.191 & 0,97 & 4 \\
\hline Arias & 7 & 1,5 & 14.610 & 1,46 & 4 \\
\hline Cali & 6 & 1,2 & 11.546 & 1,15 & 3 \\
\hline Loredo & 6 & 1,2 & 12.920 & 1,29 & 2 \\
\hline Catacora & 5 & 1,0 & 16.912 & 1,69 & 1 \\
\hline Véliz & 5 & 1,0 & 8.690 & 0,87 & 1 \\
\hline Zárate & 4. & 0,8 & 7.120 & 0,71 & 1 \\
\hline Paco & 4 & 0,8 & 3.595 & 0,36 & 2 \\
\hline Pérez & 3 & 0,6 & 9.700 & 0,94 & 1 \\
\hline Apaz & 3 & 0,6 & 3.012 & 0,30 & 2 \\
\hline Sánchez & 3 & 0,6 & 3.240 & 0,32 & 3 \\
\hline Vilca & 2 & 0,4 & 6.261 & 0,62 & 1 \\
\hline Cutipa & 2 & 0,4 & 2.410 & 0,24 & 1 \\
\hline Calane & 2 & 0,4 & 2.816 & 0,28 & 1 \\
\hline Ventura & 1 & 0,2 & 2.960 & 0,29 & 1 \\
\hline Marca & 1 & 0,2 & 1.760 & 0,17 & 1 \\
\hline Maldonado & 1 & 0,2 & 1.176 & 0,11 & 1 \\
\hline Bernabé & 1 & 0,2 & 730 & 0,07 & 1 \\
\hline Canque & 1 & 0,2 & 996 & 0,10 & 1 \\
\hline Alejandro & 1 & 0,2 & 1.406 & 0,14 & 1 \\
\hline Chusque & 1 & 0,2 & 2.230 & 0,22 & 1 \\
\hline \multicolumn{6}{|l|}{ Cifuentes } \\
\hline \multicolumn{6}{|l|}{ Mollo } \\
\hline \multicolumn{6}{|l|}{ Paredes } \\
\hline \multicolumn{6}{|l|}{ Jiménez } \\
\hline \multicolumn{6}{|l|}{ Rodríguez } \\
\hline TOTALES & 465 & 98,7 & 995.675 & 99,69 & 150 \\
\hline
\end{tabular}


Aก̃o: 1982

\begin{tabular}{|c|c|c|c|c|c|c|}
\hline$\overline{\mathbf{X}}$ sup. & Predios & $\%$ & Sup. $\left(\mathrm{m}^{2}\right)$ & $\%$ & Propietarios & $\bar{X} \sup$ \\
\hline 9.274 & 43 & 7,96 & 65.031 & 6,25 & 12 & 5.419 \\
\hline 13.811 & 66 & 12,22 & 156.541 & 5,41 & 24 & 6.522 \\
\hline 6.811 & 55 & 10,18 & 80.750 & 7,76 & 12 & 6.729 \\
\hline 5.478 & 44 & 8,14 & 71.842 & 6,94 & 18 & 3.991 \\
\hline 6.173 & 62 & 11,48 & 125.198 & 12,03 & 26 & 4.815 \\
\hline 4.944 & 48 & 8,88 & 81.940 & 7,87 & 17 & 4.820 \\
\hline 4.861 & 21 & 3,88 & 37.325 & 3,58 & 11 & 3.393 \\
\hline 5.745 & 28 & 5,18 & 53.115 & 5,13 & 9 & 5.901 \\
\hline 11.271 & 29 & 5,37 & 93.875 & 9,02 & 10 & 9.387 \\
\hline 8.712 & 6 & 1,11 & 13.056 & 1,26 & 2 & 6.528 \\
\hline 10.016 & 14 & 2,59 & 32.021 & 3,07 & 7 & 4.574 \\
\hline 5.870 & 15 & 2,77 & 22.902 & 2,20 & 10 & 2.290 \\
\hline 6.021 & 13 & 2,40 & 17.642 & 1,70 & 5 & 3.528 \\
\hline 4.451 & 8 & 1,48 & 20.790 & 1,90 & 3 & 6.930 \\
\hline 2.297 & 10 & 1,85 & 12.873 & 1,24 & 5 & 2.574 \\
\hline 3.652 & 8 & 1,48 & 14.808 & 1,43 & 3 & 4.936 \\
\hline 3.848 & 3 & 0,55 & 3.109 & 0,30 & 3 & 1.036 \\
\hline 6.460 & - & - & - & - & - & \\
\hline 16.912 & 4 & 0,74 & 15.312 & 1,48 & 3 & 5.104 \\
\hline 8.690 & 7 & 1,29 & 10.788 & 1,04 & 1 & 10.788 \\
\hline 7.120 & 4 & 0,74 & 6.180 & 0,59 & 1 & 6.180 \\
\hline 1.797 & 12 & 2,22 & 11.160 & 1,07 & 2 & 5.580 \\
\hline 9.700 & 4 & 0,74 & 10.900 & 1,05 & 1 & 10.900 \\
\hline 1.506 & 3 & 0,55 & 3.012 & 0,29 & 2 & 1.506 \\
\hline 1.080 & 8 & 1,48 & 20.529 & 1,97 & 5 & 4.105 \\
\hline 6.261 & 2 & 0,37 & 6.261 & 0,60 & 1 & 6.261 \\
\hline 2.410 & - & - & - & - & - & \\
\hline 2.816 & 3 & 0,55 & 3.536 & 0,34 & 2 & 3.268 \\
\hline 2.960 & 1 & 0,18 & 2.960 & 0,28 & 1 & 2.960 \\
\hline 1.760 & - & - & - & - & - & \\
\hline 1.176 & 8 & 1,48 & 10.897 & 1,05 & 3 & 3.632 \\
\hline 730 & 1 & 0,18 & 960 & 0,09 & 1 & 960 \\
\hline 996 & 2 & 0,37 & 2.436 & 0,24 & 1 & 2.436 \\
\hline 1.406 & 2 & 0,37 & 1.120 & 0,10 & 1 & 1.120 \\
\hline \multirow[t]{6}{*}{2.230} & - & - & - & - & - & \\
\hline & 1 & 0,18 & 25.406 & 2,45 & 1 & 25.406 \\
\hline & 1 & 0,18 & 1.020 & 0,09 & 1 & 1.020 \\
\hline & 1 & 0,18 & 280 & 0,02 & 1 & 280 \\
\hline & 2 & 0,37 & 2.410 & 0,23 & 1 & 2.410 \\
\hline & 1 & 0,18 & 2.292 & 0,22 & 1 & 2.292 \\
\hline
\end{tabular}

